PTH-045 BILIARY ASPIRATES IN SUSPECTED PANCREATIC CANCER. A USEFUL ADJUNCT TO BRUSHINGS AT ERCP?

${ }^{1}$ Duncan Napier*, ${ }^{2}$ Ben Arnold, ${ }^{1}$ Freddie Betteridge, ${ }^{1}$ Benjamin Masterman, ${ }^{1}$ Ben Colleypriest. ${ }^{1}$ Royal United Hospital, Bath, UK; ${ }^{2}$ Cheltenham General Hospital, Cheltenham, UK

\subsection{6/gutjnl-2019-BSGAbstracts.70}

Introduction NICE guidelines advocate the use of biliary brushings to obtain a tissue diagnosis in patients suspected of having pancreatic cancer when endoscopic retrograde cholangiopancreatography (ERCP) is used to relieve biliary obstruction. This retrospective audit evaluated whether biliary aspiration in addition to biliary brushings increased diagnostic yields at ERCP at a district general hospital, reducing the need for tertiary referral for endoscopic ultrasound (EUS) and fine needle aspiration (FNA).

Methods Retrospective analysis of the local endoscopy database identified all patients undergoing ERCP with biliary brushings and aspirates between July 2012 and December 2018. Histological diagnoses from biliary aspirates/brushings were compared with subsequent FNA, surgical resection or hepatobiliary multi-disciplinary team diagnosis.

Results 124 patients underwent ERCP with biliary brushings during the study period. 28 of these patients had biliary aspirates in addition to biliary brushings. Sensitivity for biliary brushings in the whole population was $47.5 \%$ (95\% CI $37.3-$ 57.8) (table 1). Specificity was 96\% (95\% CI 79.7-99.9). In the cohort undergoing biliary brushings and aspirate, sensitivity for biliary brushings alone was $23.8 \%$ (CI 8.2-47.2), whilst combination of brushings and aspirates was $28.6 \%$ (95\% CI 11.3-52.5).

Abstract PTH-045 Table 1 Cytological diagnosis (aspirate and brushings) at ERCP compared with final diagnosis

\begin{tabular}{l|c|c|c|} 
& Malignancy & Benign & Total \\
\hline $\begin{array}{l}\text { Malignant } \\
\text { Cytology }\end{array}$ & 47 & 1 & 48 \\
\hline $\begin{array}{l}\text { Benign } \\
\text { Cytology }\end{array}$ & 52 & 24 & 76 \\
\hline Total & 99 & 25 & \\
\hline
\end{tabular}

Conclusions Biliary cytology has modest sensitivity with high specificity in the detection of malignancy at ERCP. Biliary aspirate cytology increases the positive yield by 5\% compared to brushings alone and is a quick and easy technique at ERCP. Further data is required to evaluate whether biliary aspirates reliably increase the modest yield at ERCP, but this study shows a improvement from $23.8 \%$ to $28.6 \%$ in the subgroup who underwent both modalities to identify malignant cells at ERCP.

\section{PTH-046 ERCP - SHOULD WE STENT THE PANCREATIC DUCT AFTER GUIDEWIRE CANNULATION OF THE PANCREAS?}

Duncan Napier*, Benjamin Masterman, Alex Kokrashvili, Ben Colleypriest. Royal United Hospital, Bath, UK
Introduction Post ERCP pancreatitis (PEP) is a serious complication of ERCP. ${ }^{1}$ Procedural risk factors include pancreatic duct injection of contrast and pancreatic guidewire cannulation. Common bile duct (CBD) access can be challenging and pancreatic guidewire assisted cannulation (PGWAC) is an accepted technique to facilitate this. Rectal non-steroidal antiinflammatory (NSAID) administration is now widely adopted to minimise PEP in all ERCPs, whilst additional pancreatic stenting is recommended in high risk cases ${ }^{1}$, albeit with limited evidence.

Methods Retrospective data was collected from the local endoscopy database identifying all patients undergoing ERCP within 3 years. Every ERCP report was reviewed; indication, diagnosis, use of rectal NSAID and whether the CBD or pancreatic duct $(\mathrm{PD})$ was cannulated was documented. This data was then cross-referenced with hospital records to identify all patients diagnosed with pancreatitis within one-week of ERCP by reviewing medical records, biochemistry and imaging.

Results 813 ERCPs were performed in the study period; 7 were subsequently excluded due to insufficient data. The commonest indication for ERCP was choledocholithiasis (65.9\%). CBD cannulation rates were $93.56 \%$. Overall PEP rate was $2.85 \%$ (95\% CI, 1.82-4.25). The ampulla was not reached in 22 cases and thus excluded (risk of PEP deemed very low).

Of the remaining 784 patients, 107 had inadvertent PD cannulation. When the PD was cannulated, the $\mathrm{CBD}$ was successfully cannulated in 96.3\% using PGWAC, whereas when the PD was not cannulated, cannulation rate was $92.3 \%$. However in the cohort who had PD cannulation, PEP rates were statistically significantly higher $14.02 \%$ compared to those that did not have PD cannulation $(1.18 \%) \mathrm{p}<0.05$. PEP rates were $12.75 \%$ in successful PGWAC, $40 \%$ in unsuccessful PGWAC, $1.23 \%$ in successful CBD cannulation without PGWAC and $0 \%$ in unsuccessful CBD cannulation without PGWAC.

Conclusions PGWAC is an accepted technique to facilitate difficult CBD access. In the era of NSAID PEP prophylaxis for all patients the place of pancreatic stents is uncertain. Current practice for stenting the PD after PGWAC is variable. Our data would suggest that PGWAC immediately puts the patient into a high risk group for PEP and pancreatic stenting should be considered. The risk of PEP in patients where CBD cannulation is successful is $12.75 \%$ in PGWAC compared with $1.23 \%$ where the PD is not cannulated with a wire. The overall low PEP rates in this study could be potentially decreased if pancreatic stenting was employed after PGWAC and should be a further area for studies.

\section{REFERENCE}

1. Dumonceau J-M, Andriulli A, Elmunzer B J, et al. Prophylaxis of post-ERCP pancreatitis: European society of gastrointestinal endoscopy - Updated June 2014 Endoscopy 2014;46:799-815.

\section{PTH-047 OUTCOMES OF COLONOSCOPIC SURVEILLANCE AND MOLECULAR PHENOTYPING IN PATIENTS WITH FAMILY HISTORY OF COLORECTAL CANCER}

${ }^{1}$ Benjamin Norton*, ${ }^{2}$ James Coultas, ${ }^{2}$ Victoria Nicholas, ${ }^{2}$ Thomas Wright,

${ }^{1,2}$ Kevin Monahan. 'Genomic Medicine, Imperial College London, London, UK; ' ${ }^{2}$ Department of Gastroenterology, West Middlesex University Hospital, Chelsea and Westminster Hospital NHS Trust, London, UK

10.1136/gutjnl-2019-BSGAbstracts.72 
Introduction An estimated 35\% of cases of colorectal cancer (CRC) are due to heritable factors, and approximately $30 \%$ of the UK population has a family history of CRC. Those at hereditary risk should be effectively managed through registration, phenotypic and genotypic characterisation, and riskstratified colonoscopic surveillance. We assessed the impact of surveillance in patients at hereditary risk of CRC managed through the Family History of Bowel Cancer Registry at West Middlesex University Hospital (WMUH). Through analysis of this registry data, we assessed the diagnostic yield of colonoscopic surveillance and assessed the role of molecular testing.

Methods We analysed prospectively collected colonoscopic surveillance data in 361 patients undergoing surveillance at WMUH between 2010-2018. Patients were divided into five risk groups based on current BSG guidelines. Patient demographics including age, gender and family history were collated alongside colonoscopy findings and molecular data including mismatch repair (MMR) status. Impact of these variables on the prevalence of non-advanced adenomas (NAAs) and advanced adenomas (AAs) were assessed by logistic regression using SPSS software. Time to adenoma or AA was determined by survival analysis and findings were compared between index and surveillance colonoscopy.

Results In total, 640 colonoscopies were performed with 1000.1 years of follow-up. Five CRCs, 49 AAs and 170 NAAs were identified. The prevalence of CRC, AA and NAAs in patients without Lynch syndrome (LS) was $0.58 \%$, $8.19 \%$ and $26.02 \%$, respectively. Only age was significantly associated with both NAA and AA detection on multivariable analysis $(\mathrm{P}<0.05)$. Time to AA was earlier in LS patients (figure 1). A normal index colonoscopy was strongly associated with a normal finding during surveillance $(\mathrm{p}<0.001)$. In $38.6 \%$ of patients, molecular testing significantly altered surveillance strategies by recategorising familial risk.

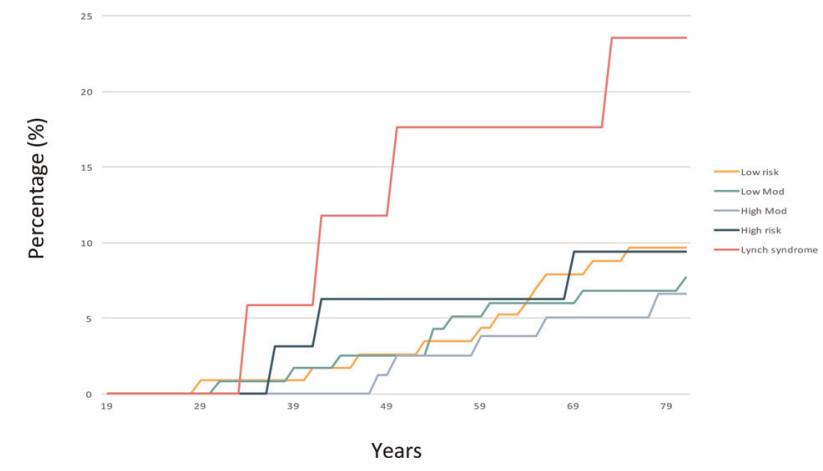

Abstract PTH-047 Figure 1 Cumulative proportion of AAs during colonoscopic surveillance according to age

Conclusion This data emphasises the strong association of colorectal neoplasia with MMR status and the need to exclude LS in patients at familial risk. Age is independently associated with colorectal neoplasia risk in this analysis, however patients often undergo colonoscopic surveillance inappropriately early. Finally, a normal index colonoscopy is associated with a low diagnostic yield in subsequent colonoscopies in this population. This low yield may influence future guideline strategies.

\section{PTH-048 COLONOSCOPIST KEY PERFORMANCE INDICATORS AND THE SURVEILLANCE OF PATIENTS WITH FAMILY HISTORY OF COLORECTAL CANCER}

${ }^{1}$ Benjamin Norton*, ${ }^{2}$ Thomas Wright, ${ }^{2}$ James Coultas, ${ }^{2}$ Victoria Nicholas,

${ }^{1,2}$ Kevin Monahan. ${ }^{1}$ Genomic Medicine, Imperial College London, London, UK; ${ }^{2}$ Department of Gastroenterology, West Middlesex University Hospital, Chelsea and Westminster Hospital NHS Trust, London, UK

\subsection{6/gutjnl-2019-BSGAbstracts.73}

Introduction Colorectal cancer (CRC) accounts for over 40,000 new cases/year in the UK, and 35\% of cases develop due to inherited susceptibility. Patients at hereditary risk undergo colonoscopic surveillance to reduce CRC incidence and mortality. High quality colonoscopy, measured by key performance indicators (KPI) including adenoma detection rate (ADR), caecal intubation rate (CIR), and polyp recovery rate (PRR) may enhance surveillance outcomes. This is important for high risk patients where colonoscopy may be a one-off intervention, or where there is significant risk of interval cancers. Consequently, we assessed colonoscopy quality in a cohort at hereditary risk of CRC managed at West Middlesex University Hospital (WMUH), and determined their relationship to colonoscopist KPIs in non-surveillance cohorts.

Methods We analysed prospective colonoscopic surveillance data through the Family History of Bowel Cancer Registry at WMUH between 2010-19. A cohort of 361 patients at hereditary risk were divided into five risk groups based on current BSG guidelines. Patient demographics including age, gender and family history were collated alongside colonoscopist data, colonoscopy findings, and KPIs. Using linear regression (SPSS software), we compared findings to ADRs, CIRs and PRRs of colonoscopists in non-surveillance patients.

Results During surveillance, 640 colonoscopies were performed by 12 clinicians. The collective ADR during index, 1st, 2nd and 3 rd surveillance colonoscopies was 18.9\%, 16.0\%, 29.6\% and $25.8 \%$, respectively. There was no difference in ADRs at Index, 1st and 2nd surveillance colonoscopy between familial risk groups. The average ADR for colonoscopists across all surveillance colonoscopies was 22.6\% (8.3-35.7\%) and CIR was $97.92 \%(50-100 \%)$. In the non-surveillance cohort ADR was 29.5\% (13.3-40.7\%), CIR 93.5\% (78.8-95.7\%) and PRR 95.2\% (86.9-98.2\%). Colonoscopist ADRs during surveillance correlated with both ADRs $\left(\mathrm{R}^{2} 0.48, \mathrm{P}<0.05\right.$; figure 1$)$ and PRRs $\left(\mathrm{R}^{2} 0.47, \mathrm{P}<0.05\right)$ in the non-surveillance cohort but not CIR.

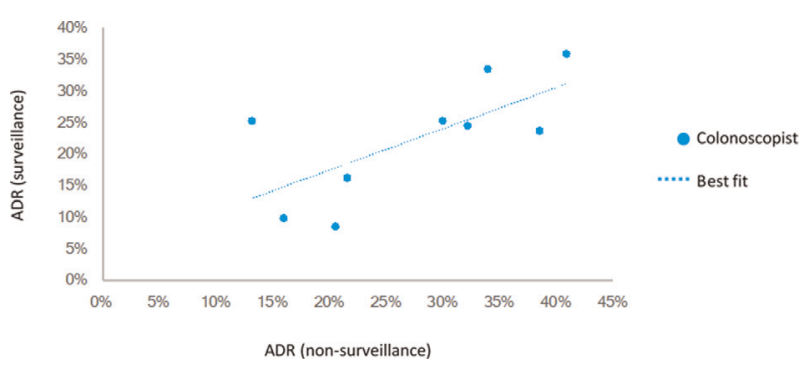

Abstract PTH-048 Figure 1 Colonoscopist ADRs in surveillance \& non-surveillance

Conclusion ADR is the main indicator of efficacy during colonoscopy and a consistent independent risk factor for interval CRCs in screening colonoscopies. Colonoscopists ADRs in 